\title{
Dental Management of Generalised Developmental Defect of Enamel in A Child with Global Developmental Delay
}

\author{
S. N. M. P. Sockalingam and H. M. Noor
}

\section{ABSTRACT}

\begin{abstract}
Development Defect of Enamel (DDE) although not common, can bring about serious sequelae and needs immediate action. If we leave the teeth untreated, many complications such as poor dental aesthetics, chipping of teeth and dental pain often set in. These complications are further aggravated if the patient has any coexisting medical issues, such as Global Developmental Delay (GDD). Children with GDD often exhibit poor oral health with an abundance of plaque that leads to caries and periodontal disease progression because of poor motor and cognitive development. Many of them also exhibit negative behaviour toward dental treatment. This case describes the dental care for a Global Developmental Delay (GDD) child with sporadic generalised Development Defect of Enamel (DDE). The case management touches on the appropriate behaviour guidance strategies used to aid the child's unfavourable behaviour towards treatment and the restorative treatment performed to preserve the structural durability of the affected teeth in a general dental setting.
\end{abstract}

Published Online: December 29, 2021

ISSN: $2684-4443$

DOI : $10.24018 /$ ejdent.2021.2.6.132

S. N. M. P. Sockalingam*

The National University of Malaysia, Malaysia.

(e-mail: drnaga67@ukm.edu.my)

H. M. Noor

Hospital Sultan Ismail, Malaysia.

(e-mail:

halimah.mohamednoor@gmail.com)

*Corresponding Author
Keywords: Behavior guidance strategies, developmental dental abnormality, enamel defect, intellectual disability.

\section{INTRODUCTION}

Global Developmental Delay (GDD) is a term coined to describe disturbances that affect two or more developmental domains such as gross or fine motor, cognition, speech, or language, social or personal, and activities of daily living [1]. The severity of GDD can vary depending on the affected domain. GDD patients often exhibit delayed progress of the various developmental domain milestones compared to their chronological peers [2]. In dentistry, GDD patients may display behavior issues such as poor cooperation for treatment, poor communication, and social adaptive skills, unable to comprehend the oral health instruction and failure to perform good mechanical plaque control because of poor hand manual dexterity. All these barriers may contribute directly or indirectly to the poor oral status and increase demand for oral healthcare in GDD patients [3]-[5]. Common oral problems seen in patients with GDD are poor oral hygiene, caries, gingivitis, halitosis, calculus, periodontal disease, and dental attrition. Gingival hyperplasia occurs if the GDD patients have coexisting epilepsy and are on antiepileptic medication [2], [5]. Besides oral problems, some GDD patients also may display varying facial features such as a broad forehead, bitemporal narrowing, up slanting palpebral fissures, low-set ears, flat nasal bridge, bulbous nose, high hard palate, cleft lip and palate, thin vermillion border and an open mouth with tongue protrusion. These features can be more profound in GDD patients with underlying genetic or syndromic aetiology [6].
Developmental Defect of Enamel (DDE) or Developmental Enamel Defect (DED) is one feature that can be seen in some GDD patients, although it is not common. DDE can occur at any stage of tooth development. Disturbances during the formative stages of a tooth, either pre-, peri- or post-natal period, can bring about quantitative or qualitative changes to the structure of the enamel [7]. The pattern of changes seen in some enamel defects may indicate the timing of the insult to the developing teeth [8]. DDE can be classified into three distinct categories, namely hypoplasia, opacity and posteruptive breakdown based on the Enamel Defect Index (EDI). These categories are further divided into few subtypes based on the pattern of the enamel defects such as demarcated or diffused opacities, pits, grooves or areas of hypoplasia and post-eruptive breakdown [9]. In children with developmental disturbance such as intellectual disability, a higher prevalence of DDE between $28 \%$ to $41 \%$ compared to healthy controls, $5 \%$ to $11 \%$ was reported [10]-[12].

DDE shows detrimental effects on the developing dentition if it is not diagnosed early. DDE can facilitate plaque adherence, thus leading to high caries susceptibility. The dental aesthetic is also a major concern in DDE because of discoloration and structural defects. Other sequelae of DDE include sensitive teeth, tooth wear, and erosion [13]. Patients with DDE may exhibit a negative association with oral health-related quality of life in the long term if the defect is not identified and treated promptly [14].

This report describes the case management of a potential cooperative young girl with GDD who has a sporadic 
generalized developmental defect of enamel. The distribution of the enamel defects was not pathognomonic to a generalized chronological DDE and was self-limiting to some surfaces of individual teeth. The management focuses on guiding the girl's apprehensive behavior to accept the planned treatment and dealing with the enamel defect, especially concerning its diagnosis, formulation of short- and long-term treatment planning. The treatment aims to preserve tooth structures, prevent further deterioration of teeth to caries, improve dental aesthetics, prevent hypersensitivity of teeth, and enhance the girl's oral health-related quality of life.

\section{Case Presentation}

A 9-year-old girl with GDD presented to the Paediatric Dental Clinic with her parents with complaints of chipped whitish front teeth and sensitive back teeth. She was a slow learner with limited communication and had difficulty in expressing herself and socializing with other children. Her gross motor development, fine motor coordination, and speech were delayed. Genetic profiling did not show any abnormalities.

Parents could not recall any history of developmental defects of the girl's primary teeth. None of her family members and immediate relatives have a history of tooth developmental defects. Her brushing technique was ineffective because of her poor motor coordination and teeth discomfort. She used children's toothpaste of 500-ppm fluoride for brushing.

She was shy, but potentially cooperative for a dental assessment. Her cognitive development was less compared to other children of her age. Although she could follow instructions and perform given tasks, she needed constant coaxing and behavior reinforcement.

The girl's extra-oral assessment showed a syndromic-like facial profile. She had a prominent forehead with a high hairline and a flat nasal bridge with slight hypertelorism. Her lips were everted, incompetent and floppy. However, no temporomandibular joint clicking, facial asymmetry, jaw deviation or limited jaw opening was noted.

Intra-oral examination showed generalized gingivitis and poor oral hygiene with a plaque score of $75 \%$. The patient presented with permanent teeth and median diastema with low thick frenum attachment extending into the interdental papilla between the maxillary permanent central incisors (teeth 11 and 21). Dental assessment of the erupted permanent teeth showed white-yellow diffuse opacities involving the cervical two-third of the labial surfaces of the maxillary permanent incisors (teeth $12,11,21,22$ ) and canine (tooth 13) with exception of the left canine (tooth 23). In the mandible, demarcated white-yellow opacities were noted on the one-third cervical aspect of the permanent lateral incisors (teeth 32 and 42) and canines (teeth 33 and 43) (Fig. 1). The palatal and lingual surfaces of the maxillary and mandibular teeth respectively were unaffected.

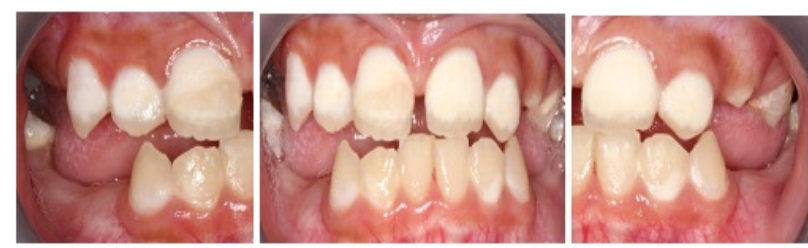

Fig. 1. Pre-treatment views of the patient's dentition.

All the first permanent molars (teeth 26, 36 and 46) except for the right maxillary first permanent molar (tooth 16) showed white-yellowish opacities. On teeth 36 and 46, the opacities were found on all surfaces. However, on tooth 26, the opacity was noted on the buccal surface only. Teeth 36 and 46 also had tooth-coloured restorations previously done at a primary health dental clinic (Fig. 2).
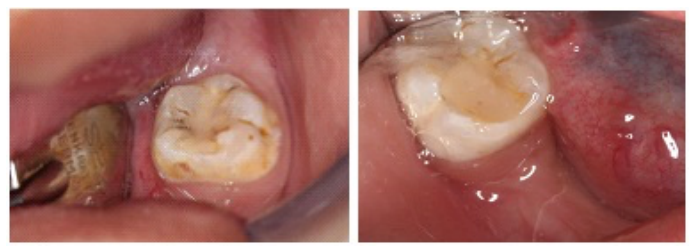

Fig. 2. Teeth 36 and 46 with DDE, defective surfaces temporised with interim GIC restoration.

Dental Panoramic Tomogram (DPT) showed the presence of all permanent teeth with impaction of the maxillary permanent second premolars (teeth 15 and 25). Radiolucent lesions were noted in the crowns of teeth 12, 11, 21 and 22. Normal radiodensity of enamel was found on all permanent molars and unerupted premolars, however, the enamel thickness appeared rather thin with spikes, especially in the premolars (Fig. 3).

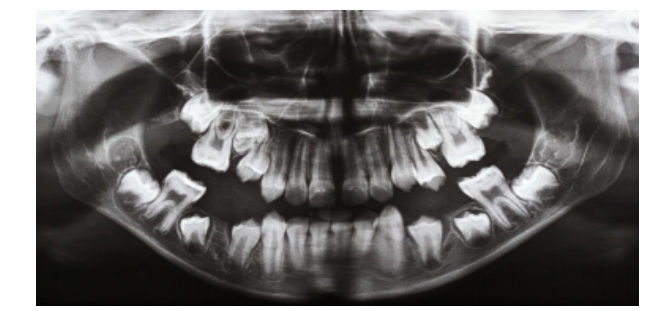

Fig. 3. Dental Panoramic Tomogram of the jaws and teeth.

Based on the clinical and radiograph findings, a diagnosis of sporadic Developmental Defects of Enamel (DDE) was made. Overall, the treatment approach focused on four key areas, namely behavior management; improvement of oral hygiene and prevention; restoration of dental aesthetics and function of the defective teeth; and continued preservation of the dental aesthetics and function from further deterioration to enable more complex restorations later in her adulthood.

Variety of behavior guidance strategies, ranging from both non-pharmacological and pharmacological behavior strategies, were used during the various treatment stages. These behavior guidance strategies helped to mould the child's potentially cooperative behavior to more cooperative behavior with time. This allowed us to perform more complex procedures. Non-pharmacological behavior guidance strategies used include direct observation, tell-show-do, positive reinforcement, and distraction. Pharmacological behavior guidance with nitrous oxide-oxygen inhalation sedation was used during the complex treatment stages. 
The cavitated defect on tooth 11 was initially temporized with glass ionomer cement, followed by reinforcement of oral hygiene and fluoride varnish application. For the definitive treatment of the maxillary and mandibular incisors and canines (teeth 12, 11, 21, 22, 13, 33, 32, 42 and 43), the opacities were removed and restored with composite resin (Filtek Z350XT ${ }^{\mathrm{TM}}$, 3M ESPE) under rubber dam isolation after infiltration of $2 \%$ mepivacaine in 1:100000 adrenaline. For the permanent first molars (teeth 26, 36 and 46) because of multi-surface opacities, the teeth were restored with indirect restorations. During the preparation of the molars, both nitrous oxide-oxygen inhalation sedation and infiltration with $4 \%$ articaine hydrochloride in 1:100000 adrenaline local anesthetic solution were used because of sensitive teeth. Impressions of the prepared teeth were taken with polyvinyl siloxane $\left(3 \mathrm{M}^{\mathrm{TM}}\right.$ ESPE Express $\left.{ }^{\mathrm{TM}}\right)$ using special trays. The prepared teeth were temporized with temporary crowns $\left(\right.$ Protemp ${ }^{\circledR}$ ) made using silicone putty moulds and cemented with Tempbond ${ }^{\circledR}$. The crowns were fabricated in the laboratory using Ceramage ${ }^{\circledR}$ composite restoration. In the subsequent visit, the fabricated composite crowns were cemented with resin-modified glass ionomer cement (Rely $\mathrm{X}^{\circledR}$ ) (Fig. 4).

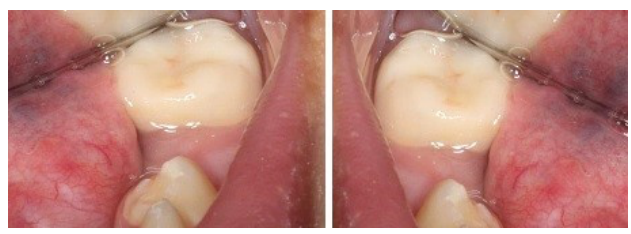

Fig. 4. Composite crowns of teeth 36 and 46.

To aid oral care, especially in the upper anterior region, a labial frenectomy was performed using a laser to release the tightness of the upper lip. This allowed easy brushing of the anterior aspect of the dentition (Fig. 5). The importance of good oral hygiene care was emphasised to the girl and her parents. They were told to pay more attention to tooth brushing, particularly at the interface between the teeth and gingiva.
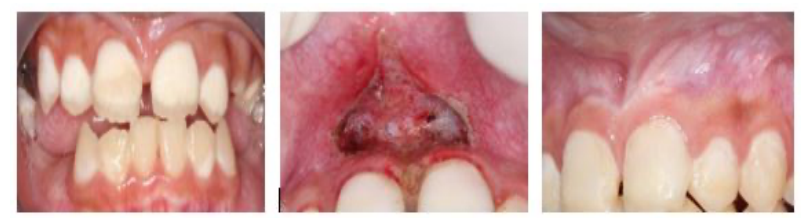

Fig. 5. Median diastema with labial frenum extending into the interdental papilla, frenectomy and 2-month post-operative view.

Six months after the completion of active treatment, the restorations were found to be intact. The patient's dental function and aesthetics showed vast improvement compared to the baseline (Fig. 6). However, her oral hygiene still required further improvement. Oral hygiene care was reinforced with dietary advice and fluoride application during the follow-up. Periodic follow-ups are necessary to assess oral hygiene improvement and to evaluate the status of the restorations as the maturation of the gingiva may expose more of the defective enamel with growth, especially at the gumteeth interfaces.

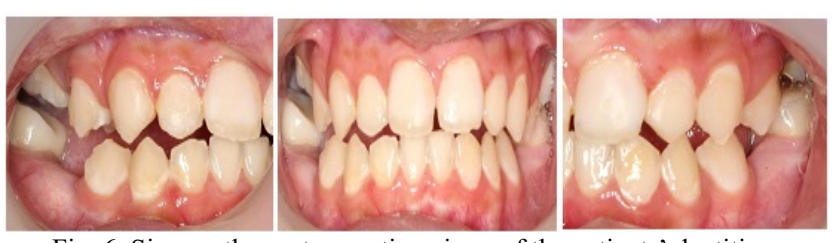

Fig. 6. Six months post-operative views of the patients' dentition.

\section{DISCUSSION}

Providing proper dental care for GDD patients, especially children in general practice settings, is often a great challenge even to pediatric dentists, what more to the general dentists [15]. Many general dentists shy away from treating GDD patients. Specialized dental services for GDD patients are often available in big hospitals and may not be accessible to all. Thus, the vast majority of GDD patients have high unmet treatment demands that may affect one's overall health and quality of life [16].

Like their healthy counterparts, GDD patients are also susceptible to oral diseases. Generally, GDD patients have poorer oral health and hygiene than those without the condition [15]. The risk of dental caries, gingivitis and periodontal disease is high in GDD patients [2]. Besides these diseases, GDD patients also exhibit other oral problems such as malocclusion, dental trauma, dental anomalies, and traumatic dental injuries [15].

Although DDE is not common compared to other oral diseases in GDD patients, its prevalence is reported higher than in healthy children [12]. In DDE, enamel formation can be affected at any phase of its development, namely the presecretory, secretory, transition or maturation phase. Disturbances during these phases can bring about either qualitative or quantitative changes to the formed enamel [17]. In the present case, the defective enamel appeared to be more of a qualitative disturbance that probably occurred either during the transition or the maturation phase of the enamel development. The distribution pattern of the enamel defects appeared generalized and non-chronological.

GDD patients exhibit a broad range of physical, intellectual, emotional, social development, diversity of temperament and attitude. There is no one tailor-made treatment protocol that fits all GDD patients [18]. Depending on the GDD severity, the use of behavior guidance strategies, either non-pharmacological or pharmacological or in combination, may help patient management [19]. This is important to ensure that quality dental care can be rendered to GDD patients. In the current case, the patient exhibited poor eye contact and limited communication with the dental team, but she was potentially cooperative. We used few types of non-pharmacological behavior guidance strategies to coax the patient into the dental operatory. At first, we used an introductory method to precondition the patient to the dental environment and the pediatric dental team. Subsequently, we used the direct observation technique to show the patient dental treatment performed on other cooperative patients to ease her fear. After the initial conditioning, she came into the operatory and sat on the dental chair but refused to open her mouth for assessment. Subsequently, we used the tell-showdo (TSD) and the verbal positive reinforcement techniques to convince the patient that dental assessment doesn't hurt. We also used a distraction method using a video monitor that 
played the patient's favorite programme during treatment. The monitor was in the patient's direct view, attached to the flexible arm of the dental chair. We were fortunate that these non-pharmacological guidance strategies were viable options for the patient. Her Frankl behavior rating scale fluctuated between score 1 (definitely negative behavior) and score 3 (positive behavior) throughout her multiple treatment sessions [20].

Plaque control is a significant aspect of oral health care in GDD patients. Many GDD patients lack physical coordination and impaired cognitive skills, therefore the ability to perform effective oral care for removal of plaque is compromised [15]. Progressive accumulation of dental plaque contributes significantly to the development of dental diseases such as dental caries, gingivitis, and periodontitis [3], [15]. In the current case, as the patient has a mild GDD, we taught the patient to take care of her oral hygiene independently, with minimal supervision and positive reinforcement from her parents. Before the commencement of each treatment session, we asked the patient to perform the oral hygiene drills of toothbrushing and flossing in the clinic. Constant advice, modification in brushing and positive reinforcement were given to enhance the patient's brushing and flossing skills because the patient is a slow learner. We also recommended the use of an electric toothbrush for better grip and effective plaque removal because of the patient's mildly impaired motor coordination. Although the patient's oral hygiene improved over time, much work is still needed to achieve the ideals. Other efforts to improve the patient's oral health include dietary analysis and counselling, and three-monthly topical fluoride application of Duraphat $\mathbb{}$ 23,600 ppm fluoride.

Dentine hypersensitivity is one of the common complaints that patients with DDE may experience. External stimuli irritate the pulp through the enamel defects that act as a poor insulator. The chronic irritation results in hypersensitivity because of easy excitement of the nerves with less stimulation. Often patients will avoid brushing the hypersensitivity teeth, thus leading to plaque accumulation and increases the risk of dental diseases with time. During restorative work, it is important to anaesthetize the defective teeth sufficiently before placing any form of restoration [21]. In the current case, we used 2\% Mepivacaine in 1:100000 adrenaline to anaesthetize all the teeth. However, we failed to achieve adequate anesthesia for the permanent molars with $2 \%$ Mepivacaine in 1:100000 adrenaline and the patient was in discomfort during to teeth preparation. The use $4 \%$ Articaine in 1: 100000 adrenaline and nitrous oxide-oxygen inhalation sedation were needed to anaesthetize the permanent molars.

Besides improving dental aesthetics and prevention of dental pain of the defective tooth structure in DDE, it is vital preserving the existing tooth structure for definitive restorations later in their adulthood. Restorative options for the affected teeth in DDE depend on the severity and extension of the lesions. For the anterior teeth, the treatments can range in ascending complexity from bleaching, microabrasion, localized composite restoration, composite veneers, and crowns. For the molars, the treatments range from fissure sealant, glass ionomer cement restoration, composite restoration to composite or stainless-steel crown
[22], [23]. In the present case, we opted for composite veneers for the anterior teeth because the enamel defects covered almost two-third of the crowns and involved the entire thickness of the enamel. For the molars, the choice of treatment was between stainless steel crowns and composite crowns to provide complete coverage of the affected teeth. Both these crowns have shown good clinical outcomes between $88 \%$ and $100 \%$ [23], [24]. Finally, we proceeded with composite crowns based on parents' preference for aesthetic restorations.

\section{CONCLuSion}

Dental practitioners often shy away from treating patients with GDD thinking that they are going to do more harm than good. Many of these patients are left with unmet dental treatment needs into adulthood. In reality, dental treatment can be carried out in general dental settings with proper assessment of the patients' conditions that will help to determine the challenges that lie ahead. Appropriate behavior guidance strategies and feasible treatment options may facilitate decent treatment fulfilment in patients with mild to moderate forms of GDD like that rendered to any healthy children as shown in the presented case.

\section{FUNDING}

Authors declare that they did not receive any funding either from internal or external organizations.

\section{CONFLICT OF INTEREST}

Authors declare that they do not have any conflict of interest.

\section{REFERENCES}

[1] Bélanger SA, Caron J. Evaluation of the child with global developmental delay and intellectual disability. Paediatr Child Health. 2018; 23(6): 403-410.

[2] Kumar S, Pai D, Saran R. Oral health characteristics and dental rehabilitation of children with global developmental delay. Case Rep Dent. 2017; 5486327.

[3] Anders PL, Davis EL. Oral health of patients with intellectual disabilities: a systematic review. Spec Care Dentist. 2010; 30(3): 110117.

[4] Zhou N, Wong HM, Wen YF, McGrath C. Oral health status of children and adolescents with intellectual disabilities: a systematic review and meta-analysis. Dev Med Child Neurol. 2017;59(10):1019-1026.

[5] Nirmala SVSG, Saikrishna D, Nuvvula S. Dental concerns of children with intellectual disability - a narrative review. Dent Oral Craniofac Res. 2018; 4(5): 1-4.

[6] Developmental delay-facial dysmorphism syndrome due to MED13L deficiency. [Internet] [cited on 2 August 2021]. Available from: https://www.orpha.net/consor/cgibin/OC Exp.php?Ing+EN\&Expert=369891.

[7] Vargas-Ferreira F, Peres MA, Dumith SC, Thomson WM, Demarco FF. Association of pre- peri- and postnatal factors with developmental defects of enamel in schoolchildren. J Clin Pediatr Dent. 2018; 42(2): 125-134.

[8] Seow WK. Clinical diagnosis of enamel defects: pitfalls and practical guidelines. Int Dent J. 1997; 47(3): 173-182.

[9] Elcock C, Lath DL, Luty JD, Gallagher MG, Abdellatif A, Bäckman $\mathrm{B}$, et al. The new enamel defects index: testing and expansion. Eur $J$ Oral Sci 2006; 114 Supp1 1: 35-38; discussion 39-41, 379. 
[10] Jindal C, Palaskar S, Kler S. The prevalence of the developmental defects of enamel in a group of 8-15 years old Indian children with developmental disturbances. J Clin Diagn Res. 2011; 5(3): 669-674.

[11] Erika V, Modrić, Verzak Ž, Karlovic Z. Developmental defects of enamel in children with intellectual disability. Acta Stomatol Croat. 2016; 50(1): 65-71.

[12] Aarthy CS, Kumar PD. Prevalence of developmental defects of enamel among children with intellectual disability - a systematic review. $J$ Global Oral Health. 2020; 3(1): 49-53.

[13] Seow WK. Developmental defects of enamel and dentine; challenges for basic science research and clinical management. Aust Dent J. 2013; 59: Suppl 1: 143-54.

[14] Andrade NS, Dos Santos IT, Lima LMS, Lima CCB, Moura LFAD, Barros SSLV, et al. Impact of developmental enamel defects on quality of life in 5-year-old children. Int J Paediatr Dent. 2019; 29(5): 557565 .

[15] National Institutes of Health, practical oral care for people with intellectual disability. Today's FDA 2010; 22(1): 55-59.

[16] Fisher K. Is there anything to smile about? A review of oral care for individuals with intellectual and developmental disabilities. Nur Res Prac. 2012; 860692.

[17] Bartlett JD. Dental enamel development: proteinases and their enamel matrix substrates. ISRN Dentistry. 2013; 24.

[18] Wilson NJ, Lin Z, Villarosa A, Lewis P, Philip P, Sumar B, et al. Countering the poor oral health of people with intellectual and developmental disability: a scoping literature review. BMC Public Health. 2019; 19: 1530

[19] American Academy of Pediatric Dentistry. Behavior guidance for the paediatric dental patient. The Reference Manual of Pediatric Dentistry. Chicago III.: American Academy of Pediatric Dentistry 2020; [Internet] [cited on 2 August 2021]. Available from: https://www.aapd.org/media/Policies_Guidelines/BP_BehavGuide.pd f.

[20] Hosey MT, Blinkhorn AS. An evaluation of four methods of assessing the behaviour of anxious child dental patients. Int $J$ Paediatr Dent. 1995; 5(2): 87-95.

[21] Almuallem Z, Busuttil-Naudi A. Molar incisor hypomineralisation (MIH) - an overview. Br Dent J. 2018; 225: 601-609.

[22] Lygidakis NA, Wong F, Jälevik B, Vierrou AM, Alaluusua S, Espelid I. Best Clinical Practice Guidance for clinicians dealing with children presenting with Molar-Incisor-Hypomineralisation (MIH) an EAPD policy document. Eur Arch Paediatr Dent. 2010; 11(2): 75-81.

[23] Koch MJ, Garcia-Godoy F. The clinical performance of laboratoryfabricated crowns placed on first permanent molars with developmental defects. J Am Dent Assoc. 2000; 131(9): 1285-1290.

[24] Discepolo K, Sultan M. Investigation of adult stainless steel crown longevity as an interim restoration in pediatric patients. Int $J$ Paediatr Dent. 2016; 27(4): 247-254. 